\title{
On a Lorentz-Invariant Interpretation of Noncommutative Space-Time and Its Implications on Noncommutative QFT
}

\section{Anca Tureanu*}

University of Helsinki and Helsinki Institute of Physics, Finland

E-mail: anca.tureanu@helsinki.fi

\begin{abstract}
By invoking the concept of twisted Poincaré symmetry of the algebra of functions on a Minkowski space-time, we demonstrate that the noncommutative space-time with the commutation relations $\left[x_{\mu}, x_{v}\right]=i \theta_{\mu \nu}$, where $\theta_{\mu \nu}$ is a constant real antisymmetric matrix, can be interpreted in a Lorentzinvariant way [1]. The implications of the twisted Poincaré symmetry on QFT on such a spacetime is briefly discussed. The presence of the twisted symmetry gives justification to all the previous treatments within NC QFT using Lorentz invariant quantities and the representations of the usual Poincaré symmetry.
\end{abstract}

International Europhysics Conference on High Energy Physics

July 21st - 27th 2005

Lisboa, Portugal

* Speaker. 


\section{Introduction}

The issue of the lack of Lorentz symmetry has been a challenge in quantum field theories on noncommutative space-time, since the field theories defined on a space-time with the commutation relation of the coordinate operators

$$
\left[\hat{x}_{\mu}, \hat{x}_{v}\right]=i \theta_{\mu v}
$$

where $\theta_{\mu v}$ is a constant antisymmetric matrix, are obviously not Lorentz-invariant, while preserving still translational invariance.

The most serious problem arises from the representation content of the subgroup of the Lorentz group, under which NC QFT is symmetric. QFT on 4-dimensional NC space-time is invariant under the $S O(1,1) \times S O(2)$ subgroup of the Lorentz group [2] (for several applications, see [3], [4], [5], [6]). However, the representation content of the $S O(1,1) \times S O(2)$ subgroup is very different from the representation content of the Lorentz group: both $S O(1,1)$ and $S O(2)$ being abelian groups, they have only one-dimensional unitary irreducible representations and thus no spinor, vector etc. representations. In this respect, one encounters a contradiction with previous calculations (for a review, see [7]), in which the representation content for the NC QFT was assumed to be the one of the Poincaré group.

The usual pretext for disregarding this contradiction was by admitting that noncommutativity should be relevant only at very short distances. Moreover, since the action of a free noncommutative field theory is the same as the action of the corresponding commutative theory, it was assumed that the Hilbert space of states is the same in the two cases, therefore noncommutativity has been often treated as a perturbation and only the corrections to first order in $\theta$ were computed. As a result, the NC QFT was practically considered Lorentz invariant in zeroth order in $\theta_{\mu \nu}$, with the first order corrections coming only from the $\star$-product.

The argument that a noncommutative free field theory is fully equivalent to its commutative correspondent has been proven false in several calculations. For example, the Casimir effect - and effect involving the energy of free fields - in the noncommutative case receives $\theta$ corrections [8]. On the other hand, it was shown that the commutators of "local" observables of free noncommutative fields are indeed different from the ones of the commutative theories and, moreover, they exhibit the residual $S O(1,1) \times S O(2)$ symmetry [9].

The solution of this contradiction has been recently given, by realizing that QFT on NC spacetime can indeed be interpreted in a Lorentz-invariant way, in the sense that the representations of the maximal symmetry algebra of NC QFT are the same as the ones of the usual QFT with Poincare invariance. This maximal symmetry is in effect a quantum symmetry, the twist-deformed (Hopf) Poincaré algebra [1] and it enables us to discuss all the aspects of relativistic QFT, which are not accessible within the residual symmetry approach [10]. For example, it justifies the attempt to prove the spin-statistics theorem in [9] in Lagrangian formulation and in [5] within the axiomatic approach.

\section{Twist deformation of the Poincaré algebra}

Usually the calculations in NC QFT are performed within the framework of the Weyl-Moyal correspondence, in which the fields are defined on the commutative counterpart of the noncom- 
mutative space-time and the usual product of fields is replaced by the Moyal $\star$-products, defined as

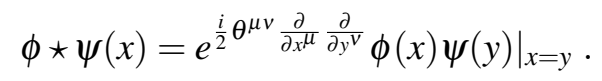

Consequently, the commutators of operators are replaced by Moyal brackets and the equivalent of (1.1) is

$$
\left[x_{\mu}, x_{v}\right]_{\star} \equiv x_{\mu} \star x_{v}-x_{v} \star x_{\mu}=i \theta_{\mu v} .
$$

The usual Poincaré algebra $\mathscr{P}$ with the generators $M_{\mu v}$ and $P_{\alpha}$ has abelian subalgebra of infinitesimal translations. Using this subalgebra it is easy to construct a twist element of the quantum group theory [11] (see also [12], [13]), which permits to deform the universal enveloping of the Poincaré algebra $\mathscr{U}(\mathscr{P})$.

This twist element $\mathscr{F} \in \mathscr{U}(\mathscr{P}) \otimes \mathscr{U}(\mathscr{P})$ does not touch the multiplication in $\mathscr{U}(\mathscr{P})$, i.e. preserves the corresponding commutation relations among $M_{\mu \nu}$ and $P_{\alpha}$,

$$
\begin{aligned}
{\left[P_{\mu}, P_{v}\right] } & =0, \\
{\left[M_{\mu v}, M_{\alpha \beta}\right] } & =-i\left(\eta_{\mu \alpha} M_{v \beta}-\eta_{\mu \beta} M_{v \alpha}-\eta_{v \alpha} M_{\mu \beta}+\eta_{v \beta} M_{\mu \alpha}\right), \\
{\left[M_{\mu v}, P_{\alpha}\right] } & =-i\left(\eta_{\mu \alpha} P_{v}-\eta_{v \alpha} P_{\mu}\right),
\end{aligned}
$$

with the essential physical implication that the representations of the algebra $\mathscr{U}(\mathscr{P})$ are the same. However, the action of $\mathscr{U}(\mathscr{P})$ in the tensor product of representations is defined by the coproduct given, in the standard case, by the symmetric map (primitive coproduct)

$$
\begin{array}{r}
\Delta_{0}: \mathscr{U}(\mathscr{P}) \rightarrow \mathscr{U}(\mathscr{P}) \otimes \mathscr{U}(\mathscr{P}) \\
\Delta_{0}(Y)=Y \otimes 1+1 \otimes Y,
\end{array}
$$

for all generators $Y \in \mathscr{P}$. The twist element $\mathscr{F}$ changes the coproduct of $\mathscr{U}(\mathscr{P})$ [11]

$$
\Delta_{0}(Y) \mapsto \Delta_{t}(Y)=\mathscr{F} \Delta_{0}(Y) \mathscr{F}^{-1} .
$$

This similarity transformation is consistent with all the properties of $\mathscr{U}(\mathscr{P})$ as a Hopf algebra if $\mathscr{F}$ satisfies the following twist equation:

$$
\mathscr{F}\left(\Delta_{0} \otimes i d\right) \mathscr{F}=\mathscr{F}\left(i d \otimes \Delta_{0}\right) \mathscr{F} .
$$

Taking the twist element in the form of an abelian twist [14],

$$
\mathscr{F}=\exp \left(\frac{i}{2} \theta^{\mu v} P_{\mu} \otimes P_{v}\right)
$$

one can check that the twist equation (2.6) is valid.

Since the generators of translations $P_{\alpha}$ are commutative, their coproduct is not deformed $\left(\Delta_{t}=\right.$ $\Delta_{0}$ is primitive)

$$
\Delta_{t}\left(P_{\alpha}\right)=\Delta_{0}\left(P_{\alpha}\right)=P_{\alpha} \otimes 1+1 \otimes P_{\alpha} .
$$

However, the coproduct of the Lorentz algebra generators is changed (see also [15]):

$$
\Delta_{t}\left(M_{\mu v}\right)=e^{\frac{i}{2} \theta^{\alpha \beta} P_{\alpha} \otimes P_{\beta}} \Delta_{0}\left(M_{\mu v}\right) e^{-\frac{i}{2} \theta^{\alpha \beta} P_{\alpha} \otimes P_{\beta}}
$$




$$
\begin{aligned}
& =M_{\mu v} \otimes 1+1 \otimes M_{\mu v}-\frac{1}{2} \theta^{\alpha \beta}\left[\left(\eta_{\alpha \mu} P_{v}-\eta_{\alpha v} P_{\mu}\right) \otimes P_{\beta}\right. \\
& \left.+P_{\alpha} \otimes\left(\eta_{\beta \mu} P_{v}-\eta_{\beta v} P_{\mu}\right)\right] .
\end{aligned}
$$

It is known (cf. [12]) that having a representation of a Hopf algebra $\mathscr{H}$ in an associative algebra $\mathscr{A}$ consistent with the coproduct $\Delta$ of $\mathscr{H}$ (a Leibniz rule)

$$
h(a \cdot b)=h_{1}(a) \cdot h_{2}(b), \quad \Delta(h)=h_{1} \otimes h_{2},
$$

the multiplication in $\mathscr{A}$ has to be changed after twisting $\mathscr{H}$. The new product of $\mathscr{A}$ consistent with the twisted coproduct $\Delta_{t}$ is defined as follows: let $\mathscr{F}=\sum f_{1} \otimes f_{2}$, then

$$
a \star b=\sum\left(\bar{f}_{1}(a)\right) \cdot\left(\bar{f}_{2}(b)\right),
$$

where $\overline{\mathscr{F}}=\sum \bar{f}_{1} \otimes \bar{f}_{2}$ denotes the representation of $\mathscr{F}^{-1}$ in $\mathscr{A} \otimes \mathscr{A}$, and the action of elements $\bar{f} \in \mathscr{H}$ on elements $a, b \in \mathscr{A}$ is the same as without twisting.

Considering the commutative algebra $\mathscr{A}$ of fields, $f(x), g(x), \ldots$, depending on the coordinates $x_{\mu}, \mu=0,1,2,3$, in the Minkowski space $M$, In $\mathscr{A}$ we have the representation of $\mathscr{U}(\mathscr{P})$ generated by the standard representation of the Poincaré algebra:

$$
P_{\mu} f(x)=i \partial_{\mu} f(x), M_{\mu v} f(x)=i\left(x_{\mu} \partial_{v}-x_{v} \partial_{\mu}\right) f(x),
$$

The Poincaré algebra acts on the Minkowski space $x_{\mu}, \mu=0,1,2,3$ with commutative multiplication:

$$
m(f(x) \otimes g(x)):=f(x) g(x) .
$$

When twisting $\mathscr{U}(\mathscr{P})$, one has to redefine the multiplication according to (2.11), while retaining the action of the generators of the Poincaré algebra on the coordinates as in (2.12):

$$
\begin{aligned}
m_{t}(f(x) \otimes g(x)) & =: f(x) \star g(x)=m \circ e^{-\frac{i}{2} \theta^{\alpha \beta} P_{\alpha} \otimes P_{\beta}}(f(x) \otimes g(x)) \\
& =m \circ e^{\frac{i}{2} \theta^{\alpha \beta} \partial_{\alpha} \otimes \partial_{\beta}}(f(x) \otimes g(x)) .
\end{aligned}
$$

Specifically, when one uses (2.14) to compute the commutator of coordinates, one obtains immediatelly:

$$
\left[x_{\mu}, x_{v}\right]_{\star}=i \theta_{\mu v}
$$

which is indeed the Moyal bracket (2.2).

\section{QFT on space-time with twisted Poincaré symmetry}

Comparing (2.1) and (2.14), it is obvious that building up the noncommutative quantum field theory through Weyl-Moyal correspondence is equivalent to the procedure of redefining the multiplication of functions, so that it is consistent with the twisted coproduct of the Poincare generators (2.8), (2.9). The QFT so obtained is invariant under the twisted Poincaré algebra. The benefit of reconsidering NC QFT in the latter approach is that it makes transparent the invariance under the twist-deformed Poincaré algebra, while the first approach highlights the violation of the Lorentz group. 
The most important feature of the QFT with twist-deformed Poincare symmetry, which deserves a special highlighting is that the representation content of the NC QFT is exactly the same as for its commutative correspondent. It is easy to see that the action of the Pauli-Ljubanski operator, $W_{\alpha}=-\frac{1}{2} \varepsilon_{\alpha \beta \gamma \delta} M^{\beta \gamma} P^{\delta}$ is not changed by the twist (due to the commutativity of the translation generators) and $P^{2}$ and $W^{2}$ retain their role of Casimir operators. Consequently, the representations of the twisted Poincaré algebra will be, just as in the commutative case, classified according to the eigenvalues of these invariant operators, $m^{2}$ and $m^{2} s(s+1)$, respectively. Besides justifying the validity of the results obtained so far in NC QFT using the representations of the Poincare algebra, this aspect will cast a new light on other closely-related fundamental issues, such as the CPT and the spin-statistics theorems in NC QFT [4, 5, 9].

\section{Conclusions}

Quantum field theory on NC space-time possesses symmetry under a twist-deformed Poincaré algebra. The twisted Poincaré symmetry exists provided that: (i) we consider $\star$-products among functions instead of the usual one and (ii) we take the proper action of generators specified by the twisted coproduct. As a byproduct with major physical implications, the representation content of NC QFT, invariant under the twist-deformed Poincare algebra, is identical to the one of the corresponding commutative theory with usual Poincaré symmetry.

\section{References}

[1] M. Chaichian, P. P. Kulish, K. Nishijima and A. Tureanu, Phys. Lett. B 604 (2004) 98, hep-th/0408069.

[2] L. Álvarez-Gaumé, J. L. F. Barbon and R. Zwicky, JHEP 0105 (2001) 057, hep-th/0103069.

[3] Y. Liao and K. Sibold, Phys. Lett. B 549 (2002) 352-361, hep-th/0209221.

[4] L. Álvarez-Gaumé and M. A. Vázquez-Mozo, Nucl. Phys. B 668 (2003) 293, hep-th/0305093.

[5] M. Chaichian, M. N. Mnatsakanova, K. Nishijima, A. Tureanu and Yu. S. Vernov, hep-th/0402212.

[6] M. Chaichian and A. Tureanu, hep-th/0403032.

[7] R. J. Szabo, Phys. Rept. 378 (2003) 207, hep-th/0109162.

[8] M. Chaichian, A. Demichev, P. Presnajder, M. M. Sheikh-Jabbari and A. Tureanu, Nucl. Phys. B 611 (2001) 383, hep-th/0101209.

[9] M. Chaichian, K. Nishijima and A. Tureanu, Phys. Lett. B 568 (2003) 146, hep-th/0209008.

[10] M. Chaichian, P. Presnajder and A. Tureanu, Phys. Rev. Lett. 94 (2005) 151602, hep-th/0409096.

[11] V. G. Drinfeld, Leningrad Math. J. 1 (1990) 321.

[12] V. Chari and A. Pressley, A Guide to Quantum Groups, Cambridge University Press, Cambridge, 1994.

[13] M. Chaichian and A. Demichev, Introduction to Quantum Groups, World Scientific, Singapore, 1996.

[14] N. Yu. Reshetikhin, Lett. Math. Phys. 20 (1990) 331.

[15] J. Wess, Deformed Coordinate Spaces Derivatives, hep-th/0408080. 